\title{
Plug-and-operate für die produktionsnahe Logistik
}

\section{Aktuelle Herausforderungen und Lösungskonzepte}

Eine flexible Automatisierung und Vernetzung produktionsnaher logistischer Anlagen und Equipments stellt die Prozessindustrie vor Herausforderungen. Im Namur-Arbeitskreis 4.19 Produktionsnahe Logistik wurden verschiedene Herausforderungen durch die Projekterfahrungen der Mitglieder identifiziert; sie werden im Beitrag vorgestellt. Ausgewählte Lösungskonzepte, die durch Überwindung klassischer Automatisierungsarchitekturen die Weiterentwicklung logistischer Produktionseinheiten und Leistungsobjekte und die Realisierung dezentral gesteuerter Einheiten ermöglichen, sind erfolgversprechend.

SCHLAGWÖRTER Industrie 4.0-Komponenten / digitaler Zwilling /

dezentrale Intelligenz / produktionsnahe Logistik

\section{Plug \& operate for production-related logistics - Current challenges and solution concepts}

Flexible automation and the interconnectedness of production-related logistics systems and equipment present the process industry with challenges. This paper presents different challenges that have been identified by members of the Namur working group 4.19 "Production-related logistics" in the course of their project work. Selected concepts are discussed that promise to overcome classic automation architectures and enable further development of logistics production units and objects as well as the implementation of decentralized units.

KEYWORDS Industry 4.0 components / digital twin / decentralized intelligence / production-related logistics 


\author{
STEPHAN KESSLER, HARTMUT LENZ, CINDY RYTIR, BAYER \\ ANNETTE SCHICK, BASF \\ MICHAEL SCHNEIDERHAN, WACKER
}

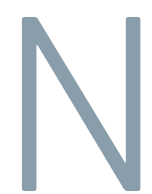

euartige Produktionskonzepte rücken verstärkt in den Fokus der Herstellungsprozesse der chemischen und pharmazeutischen Industrie. In Zukunft wird vor allem dezentralisierten Fabriken und modularen, möglichst kontinuierlich produzierenden Anlagen mit kleinen bis mittleren Kapazitätsquerschnitten eine bedeutende Rolle zugeschrieben. Dadurch müssen Produktionsabläufe und zugehörige logistische Abwicklungen auf zunehmend diversifizierte und schwankende Nachfrageverhältnisse abgestimmt und Anlagen flexibel angeordnet und miteinander verschaltet werden.

Um dies umsetzen zu können, sind die Produktionskonzepte in sichere, zuverlässige und kostengünstig zu betreibende Logistik- und Supply Chain-Prozesse einzubetten. Es ist daher erforderlich, dass die logistischen Prozesse und Systeme flexibel einsetzbar und kompatibel sind, um sie problemlos in verschiedenen Produktionsszenarien einsetzen und verschiedene logistische Objekte handhaben zu können. Die veränderten Anforderungen an die Produktion berühren somit ebenso das Design und den Betrieb von produktionsnahen Logistikprozessen, -systemen und -strukturen. Dies gilt insbesondere für die vielfältigen, in vielen Fällen weitreichend automatisierten produktionsnahen Logistikprozesse der Prozessindustrie. Idealerweise sind diese nach dem Plug-and-operate-Prinzip aufwandsarm miteinander zu verschalten.

\section{HINTERGRUND}

\section{Produktionsnahe Logistik}

Produktionslogistik, Lager- und Materialflusstechnik sind nach DIN/VDI wichtige Teile des innerbetrieblichen Leistungserstellungsprozesses, durch die sichergestellt wird, dass die richtigen Mengen an Material in der richtigen Qualität an den richtigen Stellen im Unternehmen zur richtigen Zeit bereitgestellt werden [1]. Unter produktionsnaher Logistik in der Prozessindustrie versteht der Namur-Arbeitskreis 4.19 Material- und Informationsflüsse, die der direkten Ver- und Entsorgung der chemisch-verfahrenstechnischen Transformationsprozesse (beispielsweise Synthese, Separation, Formulierung) dienen und demnach unmittelbare Schnittstellen zu diesen Prozessen haben und häufig in räumlicher Nähe zu diesen erbracht werden. Beispielhaft zu nennen sind an dieser Stelle Einwaage-, Abfüll- oder Verpackungsprozesse, aber ebenso das Puffern von Material im unmittelbaren Vor- oder Nachlauf der Produktion, zum Beispiel in Vorlagebehältern. Derartige produktionsnahe Logistikprozesse können wiederum Schnittstellen zu produktionsfernen Logistikaktivitäten aufweisen, wie Lagerung, Transport oder Umschlag von Einsatzstoffen oder Fertigwaren.

\section{Typische produktionsnahe Logistikkette}

Das in Bild 1 gezeigte Beispiel veranschaulicht anhand einer Gebindeabfüllung und -palettierung die typischen Prozessschritte eines produktionsnahen Logistikprozesses, der sich an einen verfahrenstechnischen Produktionsschritt anschließt, zum Beispiel die Herstellung von Kunststoffgranulat. Charakteristisch für diesen Prozess sind zwei Aspekte:

1 | Die Leistungsobjekte, die die einzelnen logistischen Prozessschritte durchlaufen und dabei eine Transformation erfahren; im Beispiel sind dies die abgefüllten Gebinde, die im Verlauf des Prozesses zu Ladungseinheiten gebündelt werden (Paletten mit Gebinden). Menge, Ausprägung und zeitliche Verteilung dieser Leistungsobjekte definieren die Systemlast, die das logistische System erbringen muss - im Sinne einer Transformationsleistung an den das System durchlaufenden Leistungsobjekten.

2 | Die logistischen Produktionseinheiten beziehungsweise Package Units, deren Zusammenspiel für die Erbringung der Transformationsleistung an den Leistungsobjekten sorgt; im Beispiel sind dies eine Sackabfüllanlage, verschiedene Stetigförderer für Einzelgebinde (Sack) und Ladungseinheit (Palette), Etikettierer, Palettierer und Haubenstretcher (zur Anbringung der Ladungssicherung). 


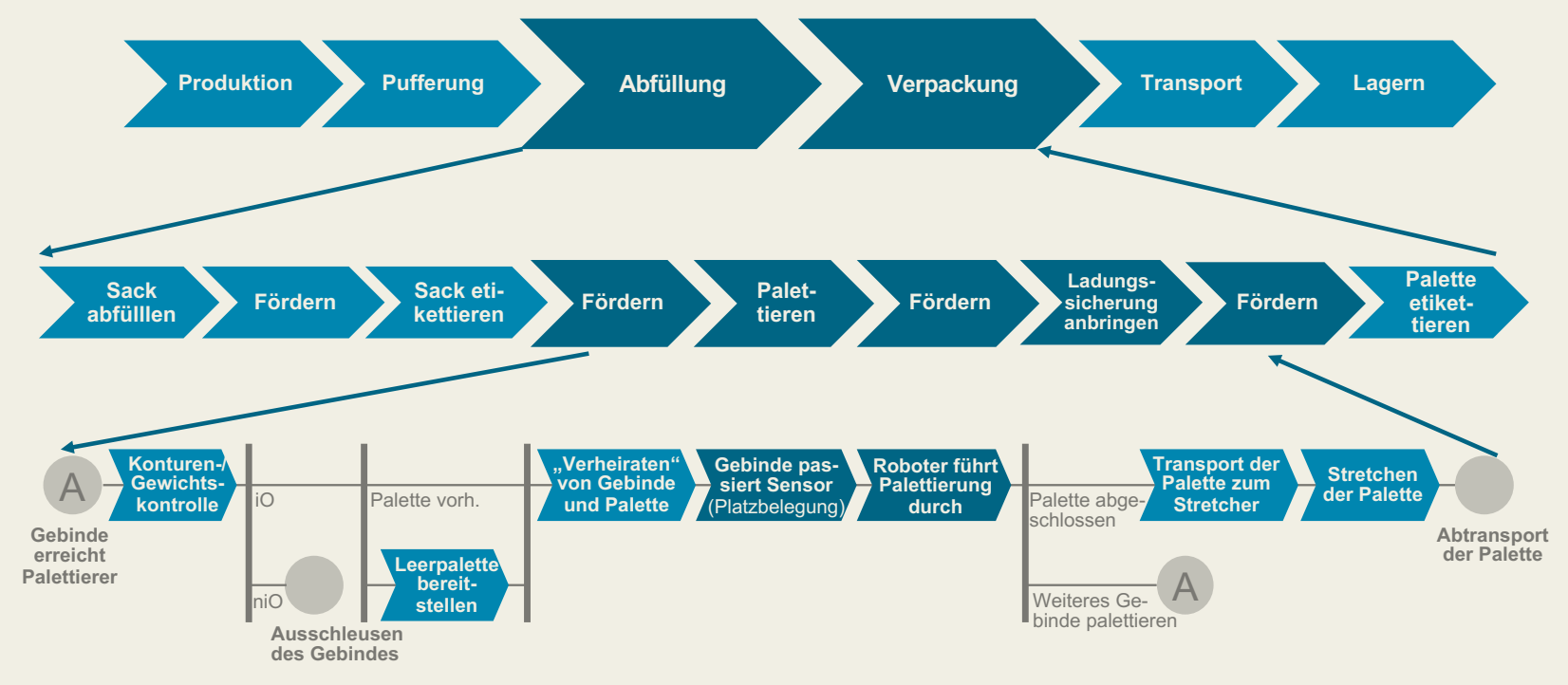

BILD 1: Prozessschritte einer typischen produktionsnahen Logistikkette

Damit die produktionsnahen Logistikprozesse den Anforderungen des Produktionsprozesses gerecht werden, ist eine materialflusstechnische Integration, aber auch ein abgestimmtes Zusammenspiel zwischen Material- und Informationsflüssen erforderlich, wie es in der untersten Ebene von Bild 1 vereinfacht für das Palettieren und Ladungssichern dargestellt wird.

\section{HERAUSFORDERUNGEN DER AUTOMATISIERUNG}

Die verschiedenen logistischen Produktionseinheiten sind möglichst flexibel miteinander und mit den zugehörigen Produktionsanlagen zu verschalten, was einerseits eine physische und andererseits eine informationstechnische Verbindung notwendig macht.

Innerhalb der Logistikautomatisierung gibt es heute mehrere, hierarchisch angeordnete Systemebenen, siehe Bild 2: Die dem physischen Materialfluss direkt überlagerte Steuerungsebene, die häufig durch speicherprogrammierbare Steuerungen (SPS) realisiert ist. Diese Ebene nimmt Detailaufgaben, wie zum Beispiel die Steuerung von Fördertechnikantrieben, wahr. Transporte und der Ressourceneinsatz werden auf der Ebene des Materialflussrechners (MFR) gesteuert. Ihm überlagert ist die verbindende Ebene der Leitsysteme angesiedelt - im Logistikkontext auch als Logistics Execution System (LES) oder Warehouse Management System (WMS) bezeichnet [2, 3]. Übergeordnete, administrative Aufgaben und Planungen werden schließlich auf der Unternehmensebene ausgeführt, in der Enterprise Resource Planning Systeme (ERP-Systeme) eingesetzt werden.
Gerade in der produktionsnahen Logistik der Prozessindustrie sind häufig hochautomatisierte Anlagen zu finden, die innerhalb der dargestellten prinzipiellen Ebenen der Logistikautomatisierung teils mit projektspezifischer Automatisierungsstruktur ausgeführt werden. So sind logistische Produktionseinheiten und -steuerungen auf die jeweiligen Prozessschritte ausgelegt und an den Produktionsprozess angepasst worden. Systemarchitekturen und Bestandssysteme sind häufig gewachsen. Bei veränderten Anforderungen an Prozesse und Anlagen müssen Schnittstellen und Programme ebenenübergreifend angepasst und Integrationskonzepte definiert werden. In Bestandsanlagen stellt die Integration neuer Anlagen beziehungsweise Komponenten im laufenden Betrieb sowohl Anwender als auch Systemhersteller vor große Herausforderungen.

Aber ebenso ist die software- beziehungsweise steuerungstechnische Integration verschiedener neuer Anlageneinheiten problematisch. Selbst einfache Operationen erfordern komplexe Verzweigungen in verschiedene Systemebenen, siehe Bild 2. Außerdem verwenden viele Hersteller proprietäre Systeme mit uneinheitlichen Schnittstellen und Semantiken, sodass Anlagenerweiterungen oder -wiederverwendungen aufwendig sind, da sich die Systeme nicht ohne Weiteres integrieren lassen. Innerbetrieblich führen diese Systeme zudem zu redundanter Datenhaltung.

Viele und insbesondere kleine, spezialisierte Hersteller von logistischen Systemen für die Prozessindustrie fokussieren sich auf die Entwicklung der Logistikhardware und unterschätzen den Validierungs- und Integrationsaufwand der IT-Systeme. Dabei gewinnt die 


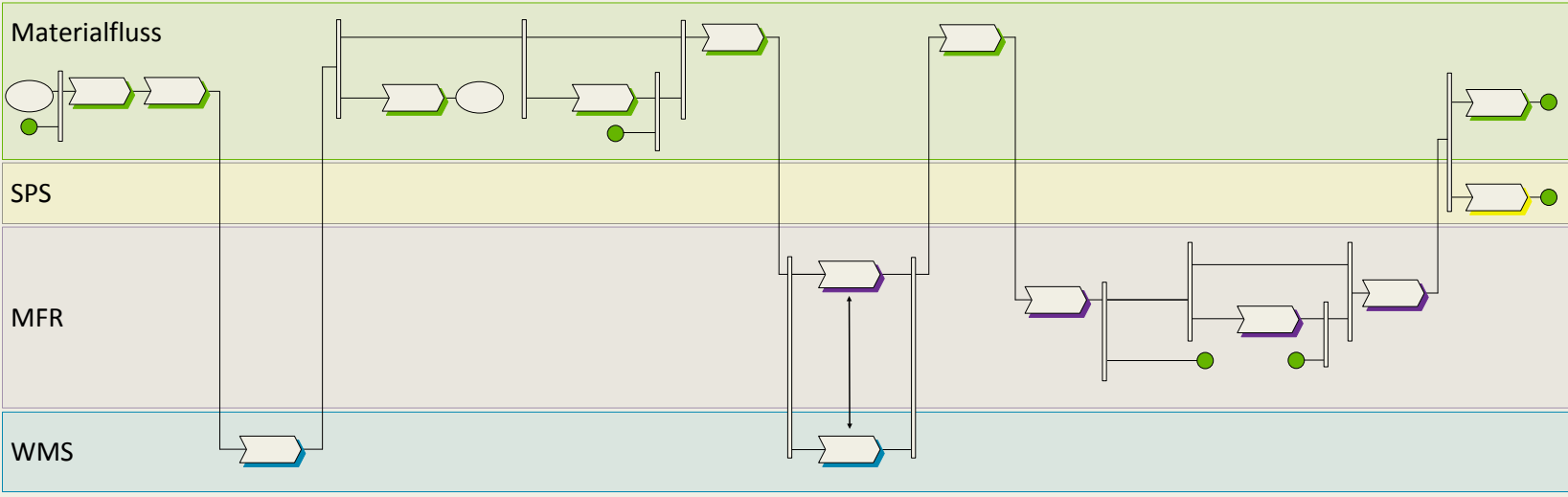

BILD 2: Schematische Darstellung eines physischen Materialflusses und begleitender IT-Prozesse

übergreifende Zusammenarbeit von Verfahrenstechnik, Automatisierung, IT und Logistik-Prozessownern bei der Prozessgestaltung in Zeiten der Digitalisierung vermehrt an Bedeutung. Eine einfachere und schnellere Integration von Systemeinheiten verspricht in diesem Zusammenhang der Industrie-4.0-Ansatz, auf den im folgenden Abschnitt eingegangen wird.

\section{INDUSTRIE-4.0-KOMPONENTEN IN DER LOGISTIK Herausforderung Digitalisierung}

Gerade im Bereich der Produktionslogistik ist im „Hinblick auf die neuen Entwicklungen zur Thematik Industrie 4.0 [...] davon auszugehen, dass zukünfige Anwendungen der Lager- und Materialflusstechnik weitgehend autonom ablaufen werden. Dazu werden Normen für elektronische Steuersysteme benötigt, um Schnittstellen definieren zu können. [...] Auch für den Datenaustausch der Geräte untereinander, z.B. zum Senden und Auswerten von Positionsdaten, müssen Schnittstellen und Protokolle definiert werden." [1]

Wie die vorstehend ausgeführten Erfahrungen mit der Integration logistischer Produktionseinheiten deutlich gemacht haben, fehlt es heute an Normen und Standards, um technische Neuentwicklungen einfach, schnell und herstellerunabhängig in bestehende Systeme integrieren zu können. Dabei spielt die Standardisierung von Logistikprozessen und -systemen sowie deren Befähigung zu autonomer Selbststeuerung im Sinne des Industrie-4.0-Paradigmas eine wichtige Rolle.

\section{Verwaltungsschale und digitaler Zwilling}

Das Konzept der cyber-physischen Systeme, zum Beispiel in Form von Industrie-4.0-Komponenten, ermöglicht hierbei Fortschritte. Industrie-4.0-Komponenten bestehen nach [4] aus einem physischen Gegenstand und einer Verwaltungsschale. Das Konzept der Verwaltungsschale zielt darauf ab, Objekte, zum Beispiel ein Produkt, ein Bauteil oder eine Maschine, im Sinne des Industrie4.0-Paradigmas kommunikationsfähig zu machen. In der Verwaltungsschale können, ähnlich wie in einer Cloud, Daten und Funktionen, die unterschiedliche Gewerke, Lebenszyklusphasen oder Analyseszenarien des Objekts betreffen, abgespeichert werden. Die Verwaltungsschale sammelt und verwaltet folglich Information zugeordneter Objekte [4].

Angenommen, verschiedene Objekte entsprechen mehreren Anlagen eines Betriebs, so dienen deren Verwaltungsschalen als Kommunikationsplattform, um Information auszutauschen. Konkret ist beispielsweise vorstellbar, dass bei einer Anlagenintegration die Soft- und Hardwareinformation der beteiligten Anlagenteile über die Verwaltungsschalen zur Verfügung gestellt und ausgetauscht wird. Dadurch entfällt eine aufwendige Anlagenintegration, wie in Abschnitt 2 beschrieben.

Ein digitaler Zwilling ist als digitales Abbild eines Objekts zu verstehen. Dem Objekt sind demnach virtuelle Daten und Information zugeordnet, die seine Eigenschaften und räumlichen Dimensionen beschreiben. Dieses Konzept stammt ursprünglich aus dem Bereich Anlagenplanung und Produktentwicklung, um verschiedene Funktionen vor der physischen Herstellung virtuell zu testen [5]. Wird dieses digitale Abbild nach Entstehung des physischen Gegenstands genutzt und begleitet diesen im Lebenszyklus, beispielsweise verfügbar gemacht über die Verwaltungsschale der Industrie-4.0-Komponente, kann es fortlaufend um relevante Information angereichert beziehungsweise erweitert werden. Mit Hilfe der digitalen Zwillinge 


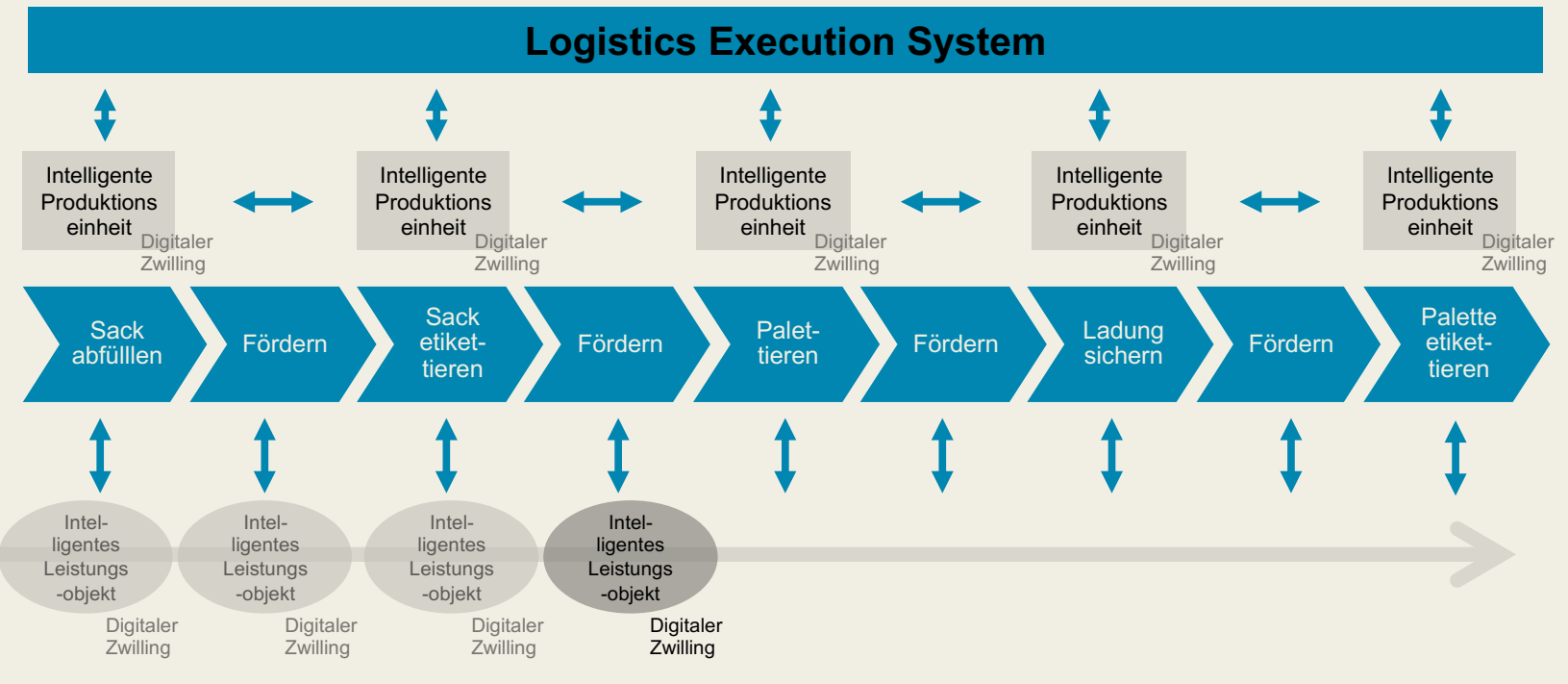

BILD 3: Plug-and-operate in der produktionsnahen Logistik durch das Zusammenspiel intelligenter Produktionseinheiten und Leistungsobjekte

ließe sich dann die Kommunikationsfähigkeit zwischen verschiedenen physischen Objekten - vergleichbar mit einer virtuellen Inbetriebnahme - vereinfacht herstellen.

Bezogen auf die eingangs beschriebene Beispielprozesskette in Bild 1 für produktionsnahe Logistik ist vorstellbar, dass sowohl die logistischen Leistungsobjekte, die diese Prozesskette durchlaufen, als auch die logistischen Produktionseinheiten als Industrie4.0-Komponenten ausgeprägt sind. Dies erlaubt die Weiterentwicklung produktionsnah eingesetzter logistischer Package Units zu intelligenten Maschinen, die flexibel miteinander verschaltet werden können und die im Zusammenspiel mit intelligenten logistischen Leistungsobjekten sowie übergeordneten Steuerungssystemen gleichzeitig für eine effiziente Leistungserbringung sorgen, siehe Bild 3.

Dies erfordert ein höheres Maß an dezentraler Intelligenz, das heißt eine Verlagerung von Daten und Steuerungsentscheidungen vom Leitsystem zu den intelligenten Produktionseinheiten beziehungweise Leistungsobjekten und ein höheres Maß der Kommunikation zwischen diesen. Die Industrie-4.0-Komponenten sind in die Lage zu versetzen, eigenständig auf äußere Einwirkungen und intern gespeicherte Vorgaben zu reagieren. In diesem Zusammenhang ist ein Automatisierungskonzept gefragt, das eine Verschaltung einzelner Einheiten ermöglicht, um diese herstellerunabhängig sowohl integrieren als auch segregieren zu können.
Erst damit rückt die Realisierung von Plug-andoperate in der produktionsnahen Logistik näher und weiterführende Industrie-4.0-Szenarien werden realisierbar, wie:

- die flexible (Re-)Konfiguration einzelner Prozessschritte und die Integration neuer Module in eine Gesamtanlage (vergleiche [6], Ausführung zur Seifenanlage der SmartFactory ${ }^{\mathrm{KL}}$ ),

- die autonome Selbstadaption, die einer eigenständigen Anpassung eines Systems aus intelligenten Produktionseinheiten beziehungsweise Leistungsobjekten an eine neue Produktionsaufgabe entspricht, vergleiche [7],

- oder die begleitende Selbstoptimierung miteinander verketteter intelligenter Produktionseinheiten im Betrieb, vergleiche [4].

\section{LÖSUNGSKONZEPTE}

In der Namur, aber ebenso innerhalb anderer Initiativen wurden bereits erste Konzepte erarbeitet, um die Modularisierung von Anlagen - auch vor dem Hintergrund der automatisierungstechnischen Umsetzung zu unterstützen. Der Namur-AK 4.19 versteht sich als Plattform, um die Anwendung dieser Lösungskonzepte im Kontext der Logistikautomatisierung in der Prozessindustrie zu betrachten. Nachfolgend werden daher drei vielversprechende Lösungskonzepte vorgestellt und hinsichtlich ihrer Relevanz für das Arbeitsfeld des Arbeitskreises beleuchtet. 


\section{Namur-Modul-Type-Package}

Durch die Herausgabe der Namur-Empfehlung „Anforderungen an die Automatisierungstechnik durch die Modularisierung verfahrenstechnischer Anlagen“ (NE 148) wurden notwendige Entwicklungen und Rahmenbedingungen für die automatisierungstechnische Integration mehrerer Module zu einer Gesamtanlage aufgezeigt [8]. Das Automatisierungskonzept Dima (Dezentrale Intelligenz Modularer Anlagen) setzt die Anforderungen an die Systemarchitektur aus der Namur-Empfehlung erstmals um [9].

Das gemeinsam von Namur und ZVEI entwickelte Modul Type Package, kurz MTP, knüpft daran an. Alle Information, die für eine Modulintegration in die Prozessführungsebene benötigt wird, enthält demnach das Modul selbst. Durch das MTP wird ein Modul herstellerneutral beschrieben, Workflows zur projektunabhängigen Definition der Module und ihre projektbezogene, automatisierungstechnische Integration werden vorgegeben und die Entwicklung von Engineering-Werkzeugen zum Export und Import des MTP wird angestoßen. Neben der physikalischen Modularisierung stellt das Modul seine automatisierungstechnische Funktionalität über das MTP als Dienst der übergeordneten Prozessführungsebene zur Verfügung.

Einem agilen Entwicklungsansatz folgend wurde auf der Namur-Hauptversammlung 2016 ein MTP-Prototyp vorgestellt. Am Beispiel der Integration der MenschMaschine-Schnittstelle konnte aufgezeigt werden, dass verfahrenstechnische Module mit diesem Ansatz grundsätzlich herstellerunabhängig in die Prozessführungsebene eingebunden und zu einer Gesamtanlage zusammengeführt werden können [10, 11].

Da die Realisierung von Plug-and-operate in der produktionsnahen Logistik ebenfalls eine herstellerneutrale automatisierungstechnische Integration verschiedener logistischer Produktionseinheiten zum Ziel hat, erscheint die Prüfung der Nutzbarkeit oder vielmehr Erweiterbarkeit des MTP-Ansatzes auch für dieses Anwendungsfeld erstrebenswert. Dabei wird zu klären sein, inwiefern über MTP auch das in Bild 3 dargestellte Zusammenspiel logistischer Package Units mit intelligenten logistischen Leistungsobjekten realisierbar ist. Auch die verstärkt erwartete, direkte Machine-to-Machine-Kommunikation zwischen den einzelnen logistischen Anlagenbausteinen ist im heutigen Entwicklungsstand des MTP noch weitgehend unberücksichtigt, da die Module in eine übergeordnete Steuerungsebene, wie das Prozessleitsystem eingebunden sind und die Funktionen dort gebündelt und abgerufen werden.

\section{Namur Open Architecture}

Insbesondere am zuletzt genannten Punkt setzt die Namur Open Architecture (NOA) an. Sie stellt eine Erweiterung beziehungsweise eine Adaptionsmöglichkeit der klassischen Automatisierungspyramide für zusätzliche Funktionen bereit, siehe Bild 4 [12]. Zu NOA finden sich zwei weitere Aufsätze in dieser Ausgabe.

Bestehende hierarchische Automatisierungsstrukturen sollen gezielt und kontrolliert geöffnet und so ergänzt werden, dass Möglichkeiten von Industrie 4.0, wie die Integration schnelllebiger IT-Komponenten im Rahmen des Internet of Things, nutzbar werden. Durch die offenere Systemstruktur mit NOA können zusätzliche Sensoren für Monitoring- und Optimierungsprozesse einfach integriert und parallel dazu vorhandene Information durch die Kern-Automatisierung an bestehenden Feldgeräten abgeholt werden. Der NOA-Ansatz kann somit als offener Kommunikationsstandard für die Erstellung digitaler Zwillinge für komplette Funktionseinheiten aufgefasst werden.

Wie Abschnitt 2 dieses Artikels deutlich gemacht hat, fehlt bei der Automatisierung produktionsnaher Logistikprozesse ein herstellerübergreifend anerkannter Standard, der systemunabhängige Schnittstellen und Protokolle vorgibt. Die automatisierungstechnische Einbindung intelligenter logistischer Leistungsobjekte, die sich häufig nur für einen begrenzten Zeitraum im betrachteten System befinden, erfordert zudem die Berücksichtigung von Information aus vergleichsweise kurzlebigen informationstechnischen Komponenten. Eine Forcierung der Standardisierungsbemühungen von Anwenderseite durch die Nutzung von NOA als geeignetem Kommunikationsstandard kann hier sicherlich unterstützend wirken und ebenso im Logistikkontext Anwendung finden.

In Bezug auf die produktionsnahe Logistik ist die Nutzung von NOA für Zusatzfunktionen denkbar, beispielsweise bei Störungen. Fällt eine Anlage, wie der Palettierer im in Bild 3 dargestellten Prozess aus, könnte über eine Zusatzfunktion, die nicht durch die

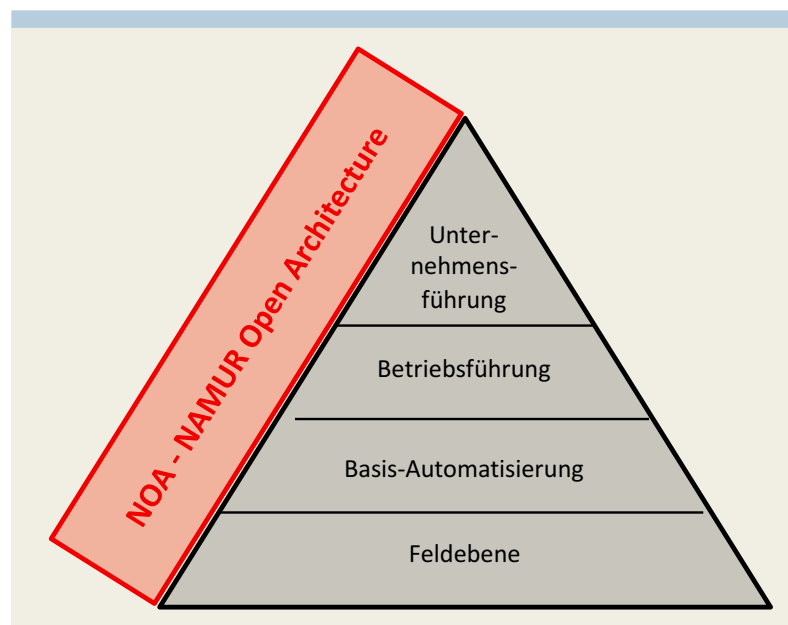

BILD 4: Erweiterung der klassischen Automatisierungspyramide durch Namur Open Architecture [12] 
Kernautomatisierung abgebildet ist, reagiert werden. Vorgeschaltete Anlagen, wie die Abfüllanlage, können ihre Geschwindigkeit gezielt anpassen, um den Problemen am Palletierer entgegenzuwirken.

\section{Smart Factory}

Ein anderes Lösungskonzept, das für die Modularisierung und automatisierungstechnische Integration von Anlagen eingesetzt werden kann, wurde in der Stückgutindustrie unter dem Begriff Smart Factory bereits in Testanlagen umgesetzt [6]. Die Smart Factory verbindet dabei mehrere Ideen von Industrie 4.0.

Die Grundidee besteht darin, eine Fabrik zu gestalten, die sich dezentral und echtzeitnah selbst steuern kann Unter dem Begriff smart wird in diesem Zusammenhang die Fähigkeit verstanden, aufgrund eigener Informationsverarbeitung mit der Umwelt Information austauschen und anspruchsvolle Funktionalitäten anbieten zu können [13]. Folglich besteht die Möglichkeit, dass Material, Maschinen, Lager- und Logistiksysteme miteinander kommunizieren können und damit jeder Zustand der Wertschöpfungskette genau beschreibbar ist. Dies lässt sich zur Steuerung eines möglichst optimalen Wertstroms nutzen. Werden die Überlegungen der Smart Factory auf die klassische Automatisierungspyramide aus Bild 4 oder die in Abschnitt 2 angesprochenen Systemebenen der Logistikautomatisierung angewandt, werden Entscheidungen nicht mehr an der Spitze der Pyramide getroffen. Stattdessen können verbundene Komponenten beziehungsweise Systemteile ihre Dienste zur Verfügung stellen und direkt untereinander - auf unterster Ebene der Automatisierungspyramide - austauschen [14].

In Demonstrationsanlagen zur Smart Factory wurden Anlagenteile unterschiedlicher Hersteller flexibel zu Produktionsanlagen kombiniert, die beispielsweise über intelligente Werkstückträger gezielt auch Information über das zu transformierende Leistungsobjekt für die Prozesssteuerung nutzen. Ein Factory Backbone sorgt

\section{REFERENZEN}

[1] DIN, VDI (2015). Deutsche Normungsroadmap Logistik. Berlin/Düsseldorf: VDI, S. 12, S. 20, S. 28

[2] Kilger, C. und Herman, R. (2006). Integriertes Informationsmanagement in Intralogistik-Systemen - Architektur und Potenzial. In: D. Arnold, ed., Intralogistik. Potentiale, Perspektiven, Prognosen. Berlin/Heidelberg: Springer-Verlag, S. 212-223

[3] VDI 3601 (2015). Warehouse-Management-Systeme. http://www.vdi.de

[4] BMWi Bundesministerium für Wirtschaft und Energie (2016). Ergebnispapier Plattform Industrie 4.0. Struktur der Verwaltungsschale - Fortentwicklung des Referenzmodells für die Industrie 4.0-Komponente, S. $11 \mathrm{ff} .$, S. $19 \mathrm{ff}$

[5] Geipel-Kern, A. (2016). Auf dem Weg zur digitalen Reife. Process - Chemie-Pharma-Verfahrenstechnik, 23(10), S. 34-35

[6] Intelligent, modular - und bereits in industrietauglicher Ausführung: Die Industrie 4.0-Anlage von SmartFactory ${ }^{\mathrm{KL}}$.[online] SmartFactory ${ }^{\mathrm{KL}}$. Verfügbar unter: http://www.smartfactory.de/

[7] Vogel-Heuser, B. (2014). Herausforderungen und Anforderungen aus Sicht der IT und Automatisierungstechnik. In: T. Bauernhansl, ed., Industrie 4.0 in Produktion, Automatisierung und Logistik. Wiesbaden: Springer Fachmedien, S. 42-45

[8] NE 148 (2013). Anforderungen an die Automatisierungstechnik durch die Modularisierung verfahrenstechnischer Anlagen. http://www.namur.org
[9] Bernshausen, J., Haller, A., Holm, T., Hoernicke, M., Obst, M. und Ladiges, J. (2016). Namur Modul Type Package - Definition. Beschreibungsmittel für die Automation modularer Anlagen. atp edition, 58(1-2), S. 72-81

[10] Bernshausen, J., Haller, A., Holm, T., Hoernicke, M., Obst, M. und Ladiges, J. (2016). Namur Modul Type PackageDefinition. atp edition, 58(1-2), S. 72-81

[11] Holm, T., Obst, J., Ladiges, L., Urbas, L., Fay, A., Albers, T. und Hempen, U. (2016). Namur Modul Type Package - Implementierung. Anwendung des Namur-MTP für Prozessanlagen. atp edition, 58(1-2), S. 82-90

[12] Klettner, C., Tauchnitz, T., Epple, U., Nothdurft, L., Diedrich, C., Schröder, T., Großmann, D., Banerjee, S., Urbas, L. und latrou, C. (2016). NOA - Namur Open Architecture, atp edition, 59(1-2), S. 20-37

[13] Acatech (2014). Intelligente Objekte - Klein, vernetzt, sensitiv. Eine neue Technologie verändert die Gesellschaft und fordert zur Gestaltung heraus. Acatech bezieht Position 5, S. 9

[14] Kleinemeier, M. (2014). Von der Automatisierungspyramide zu Unternehmenssteuerungsnetzwerken. In: T. Bauernhansl, ed., Industrie 4.0 in Produktion, Automatisierung und Logistik.Wiesbaden: Springer Fachmedien, S. 571-579

[15] Fischer, S. und Gorecky, D. (2016). Smart Factory - Eine Idee wird Realität. In: R. Heinze, C. Manzei, L. Schleupner, ed., Industrie 4.0 im internationalen Kontext. Kernkonzepte, Ergebnisse, Trends. Berlin/Offenbach: VDE Verlag, S. 152-154 


\section{DANKSAGUNG}

Der Inhalt dieses Beitrags basiert überwiegend auf Erfahrungen und Information der Mitglieder des Namur-Arbeitskreises 4.19 und greift darüber hinaus auf Materialien beziehungsweise Beiträge anderer Namur-Arbeitskreise zurück. Daher gilt der Dank dem offenen Austausch im Arbeitskreis und dem Zugang zu Information, die durch die Plattform der Namur zugänglich ist.

für eine einheitliche Infrastruktur, da Anlagenteile über eine einheitliche Schnittstelle verfügen und über diese angebunden werden können [15].

Bisherige Überlegungen und Anwendungen zur Smart Factory fokussieren nicht die Prozessindustrie und dort ablaufende produktionsnahe Logistikprozesse. Entsprechend dem in Bild 3 dargestellten Ansatz, ist die in der Smart Factory angestrebte Vernetzung und der direkte Informationsaustausch zwischen intelligenten Leistungsobjekten und den intelligenten Produktionseinheiten vielversprechend. Schließlich spielt das Handling von Stückgut - im Sinne von in Gebinden und/oder auf Ladungsträgern verpackten Materialien - auch in produktionsnahen Logistikketten der Prozessindustrie eine große Rolle.

\section{FAZIT}

Um der Realisierung von Plug-and-operate in der produktionsnahen Logistik näher zu kommen, sind intelligente, echtzeitorientierte und standardisierte Bausteine (im Sinne von logistischen Produktionseinheiten) erforderlich, die herstellerunabhängig, wiederverwertbar, wandlungsfähig und modular je nach Prozessanforderung unter Nutzung der technologischen und digitalen Möglichkeiten miteinander vernetzt werden können. Diese müssen eine wirtschaftliche, sprich minimale Gesamtdurchlaufzeit aller Aufträge bei optimierten Beständen sicherstellen und zwar nicht nur bereichsbezogen, sondern möglichst auch unternehmensübergreifend. Nur so lässt sich die Idee umsetzen, kostengünstig neue Anlagenteile in bestehende Anlagen zu integrieren beziehungsweise neue Anlagen schneller aus Modulen zu erstellen.

Gewährleistet werden kann dies durch einheitliche IT- und Prozess-Standards sowie durch eine enge Kooperation zwischen Anlagen-, Fördertechnik- sowie Software-Herstellern. Erst das Zusammenspiel und gemeinsame Gestalten von Prozessen durch Verfahrenstechnik, Automatisierung, IT und Logistik macht Plug-and-operate möglich.

MANUSKRIPTEINGANG 22.12.2016

\section{AUTOREN}

Dr.-Ing. STEPHAN KESSLER (geb. 1977) ist als Lead Discipline Engineer für Logistics Engineering und Consulting im Bereich Engineering und Technology der Bayer AG tätig. In dieser Funktion entwickelt und implementiert er logistische Konzepte für Standorte und Produktionsanlagen der Prozessindustrie.

Dipl.-Ing. HARTMUT LENZ (geb. 1964) leitet als Director das Team Logistics Engineering \& Consulting im Bereich Engineering und Technology der Bayer AG. Gemeinsam mit seinem Team stellt er fachliche Expertise im Bereich Logistik für die Bayer AG bereit und realisiert Projekte weltweit.

Bayer AG, Namur AK 4.19,

Kaiser-Wilhelm-Allee, Gebäude K9,

51368 Leverkusen,

Tel. +49 (0) 2143057611 ,

E-Mail: hartmut.lenz@bayer.com
CINDY RYTIR (geb. 1993) ist Studentin im Studiengang Wirtschaftsingenieurwesen der Hochschule Mannheim und verfasst ihre Abschlussarbeit im Bereich Engineering und Technology der Bayer AG.

Dr. ANNETTE SCHICK (geb. 1967) ist als Process Designer für Manufacturing und Site Logistics Prozesse im Bereich Information Services \& Supply Chain Operations der BASF tätig. In dieser Funktion entwickelt sie als Teil der globalen Business Process Management Abteilung Prozesse und Strategien im Bereich Produktion und Site Logistics.

Dipl.-Kfm. MICHAEL SCHNEIDERHAN (geb. 1961) ist als Direktor Information Technology für die Themengebiete Factory Applications innerhalb der Wacker Chemie AG zuständig. In dieser Funktion konzipiert, entwickelt und betreibt er IT Lösungen beziehungsweise Applikationen im Umfeld von Manufacturing und Logistics, Laboratory und Process Data Management. 